\title{
Hinode observations reveal boundary layers of magnetic elements in the solar photosphere
}

\author{
R. Rezaei ${ }^{1}$, O. Steiner ${ }^{1}$, S. Wedemeyer-Böhm ${ }^{2}$, R. Schlichenmaier ${ }^{1}$, W. Schmidt ${ }^{1}$, and B. W. Lites ${ }^{3}$ \\ 1 Kiepenheuer-Institut für Sonnenphysik, Schöneckstrasse 6, 79104 Freiburg, Germany \\ e-mail: [rrezaei;steiner;schliche;wolfgang]@kis.uni-freiburg.de \\ 2 Institute of Theoretical Astrophysics, PO Box 1029, Blindern, 0316 Oslo, Norway \\ e-mail: sven.wedermeyer-bohm@astro.uio.no \\ ${ }^{3}$ High Altitude Observatory, NCAR, PO Box 3000, Boulder, CO 80307, USA \\ e-mail: lites@ucar.edu \\ Received 27 July 2007 / Accepted 31 October 2007
}

\section{ABSTRACT}

\begin{abstract}
Aims. We study the structure of the magnetic elements in network-cell interiors. Methods. A quiet Sun area close to the disc centre was observed with the spectro-polarimeter of the Solar Optical Telescope on board the Hinode space mission, which yielded the best spatial resolution ever achieved in polarimetric data of the Fe I $630 \mathrm{~nm}$ line pair. For comparison and interpretation, we synthesize a similar data set from a three-dimensional magneto-hydrodynamic simulation.

Results. We find several examples of magnetic elements, either roundish (tube) or elongated (sheet), which show a central area of negative Stokes- $V$ area asymmetry framed or surrounded by a peripheral area with larger positive asymmetry. This pattern was predicted some eight years ago on the basis of numerical simulations. Here, we observationally confirm its existence for the first time. Conclusions. We gather convincing evidence that this pattern of Stokes- $V$ area asymmetry is caused by the funnel-shaped boundary of magnetic elements that separates the flux concentration from the weak-field environment. On this basis, we conclude that electric current sheets induced by such magnetic boundary layers are common in the photosphere.
\end{abstract}

Key words. Sun: photosphere - Sun: magnetic fields

\section{Introduction}

With ever increasing polarimetric sensitivity and spatial resolution, it becomes now clear that even the most quiescent areas on the solar surface harbour ample amounts of magnetic flux. This flux becomes visible in small patches of field concentrations, called magnetic elements for short. While horizontal fields tend to occur near the edge of granules, the present investigation focusses on fields predominantly oriented in the vertical direction and mainly occurring in the intergranular space (Lites et al. $2007 \mathrm{~b}, \mathrm{a})$. There, magnetic elements are often visible in G-band filtergrams as point-like objects. In more active regions, magnetic elements of extended size appear in the form of "crinkles", "ribbon bands", "flowers", etc. (Berger et al. 2004), which show a sub-structure consisting of a dark central area surrounded by a striation of bright material and further outside by a downdraft of plasma (Langangen et al. 2007). While Doppler measurements of this downdraft are at the limit of spatial resolution with the best ground-based solar telescopes, spectro-polarimetric measurements have less spatial resolution due to image degradation by atmospheric seeing over the required long integration times.

Measurements with the spectro-polarimeter on board the Hinode satellite have a superior spatial resolution of approximately $0.3^{\prime \prime}$ thanks to excellent pointing capabilities (Shimizu et al. 2007) and the absence of seeing. Using Hinode data, we show examples of unipolar magnetic elements of the networkcell interiors that possess a distinct sub-structure, which is strikingly manifested in maps of the asymmetry of spectral lines in the circularly polarized light - the Stokes- $V$ area asymmetry.
The areas of the blue and the red lobes of Stokes- $V$ profiles are identical when formed in a static atmosphere, but become asymmetric in the presence of gradients in the velocity and magnetic field strength (Illing et al. 1975; Solanki \& Pahlke 1988; Sanchez Almeida et al. 1989; Landolfi \& Landi degl'Innocenti 1996). Hence, the variation in the Stokes- $V$ asymmetry across magnetic elements gives information on their magnetic field and plasma flow properties. Since this information is involved, we computed synthetic Stokes- $V$ profiles of magnetic elements that occur in a three-dimensional simulation of magneto-convection and compare their asymmetry with the measured ones in order to understand their origin.

\section{Observation and data reduction}

The observations were carried out on 10 March, 2007 with the spectro-polarimeter (SP) of the Solar Optical Telescope (SOT) on board of the Hinode space satellite (Kosugi et al. 2007; Tsuneta et al. 2007; Suematsu et al. 2007). The four Stokes profiles of the Fe I $630 \mathrm{~nm}$ line pair were recorded in a quiet Sun area close to the disc centre. The integration time of $4.8 \mathrm{~s}$ led to an rms noise level in the polarization signal of $\sigma \approx 1.2 \times 10^{-3} I_{\mathrm{c}}$. The data calibration for the SP is described in Ichimoto et al. (2007) and Tarbell (2006). The field of view comprises an area of $162^{\prime \prime} \times 302^{\prime \prime}$ with a spatial sampling of $0.16^{\prime \prime}$ along the slit and $0.15^{\prime \prime}$ in the scanning direction. The spatial resolution of the resulting spectro-polarimetric maps is approximately $0.3^{\prime \prime}$, and the spectral sampling is $2.15 \mathrm{pm}$. The rms continuum contrast at $630.0 \mathrm{~nm}$ is around $7.5 \%$, far above the $3 \%$ value typically 


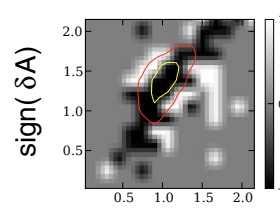

$>\underbrace{\infty}_{1,5}$
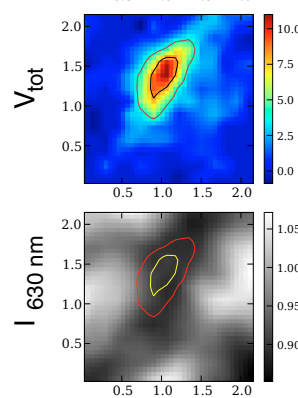

b
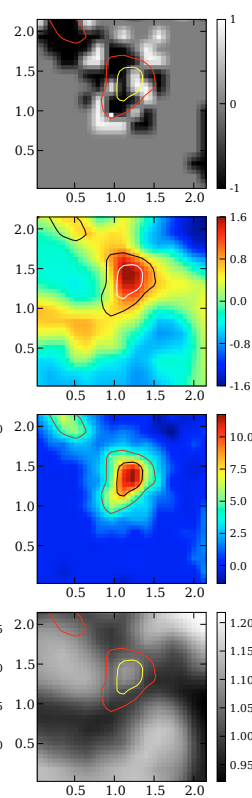

c
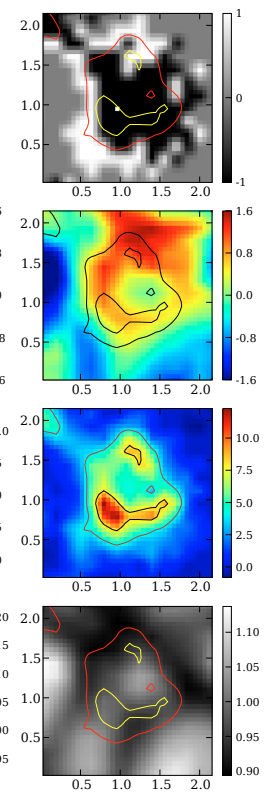

d
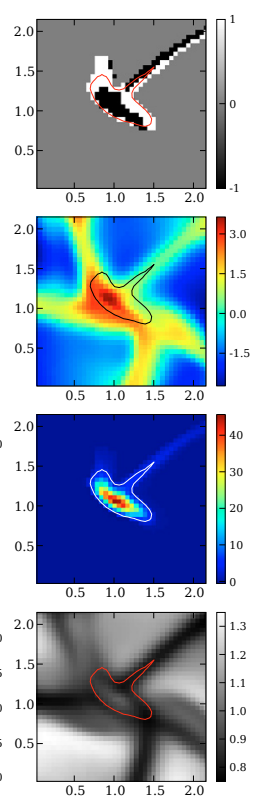

e
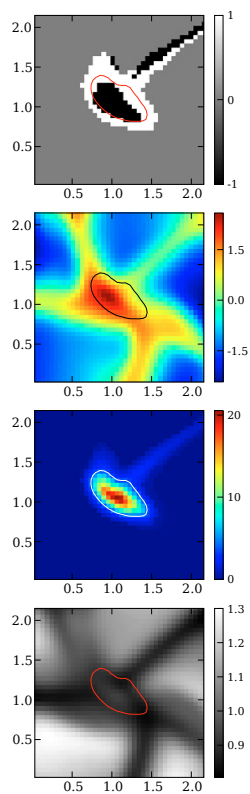

f
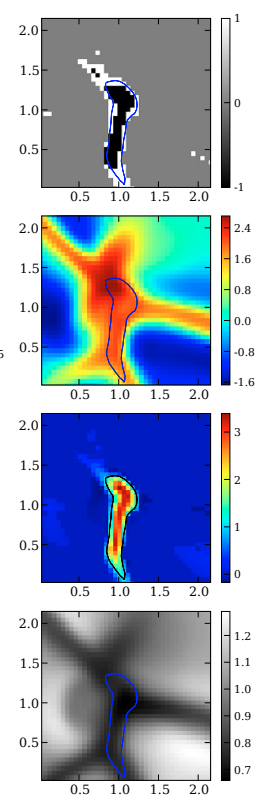

g
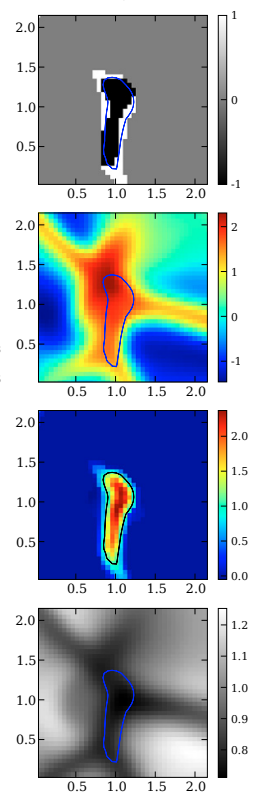

Fig. 1. Columns a)-c): observational data obtained with the spectro-polarimeter of Hinode/SOT. Columns d) and f): synthetic data from the $3 \mathrm{D}$ MHD simulation. Columns $\mathbf{e}$ ) and $\mathbf{g}$ ): same as d) and f) but after application of the SOT-PSF to the synthetic intensity maps. Rows from top to bottom: the sign of the Stokes- $V$ area asymmetry for the line Fe I $630.25 \mathrm{~nm}$, where black and white correspond to negative and positive, respectively, the Fe I $630.15 \mathrm{~nm}$ line-wing velocity in $\mathrm{km} \mathrm{s}^{-1}, V_{\text {tot }}$ of Fe I $630.25 \mathrm{~nm}$ in $\mathrm{m} \AA$, and the continuum intensity at $630.0 \mathrm{~nm}$. Contours refer to $V_{\text {tot }}$. Distance between tick marks is $0.5^{\prime \prime}$.

observed from the ground at the same wavelength (Rezaei et al. 2007; Khomenko et al. 2005). This same data set served Lites et al. $(2007 \mathrm{a}, \mathrm{b})$ in an investigation of the horizontal magnetic fields in the quiet Sun.

\section{Numerical simulation}

The three-dimensional magnetohydrodynamic simulation comprises an area of $4800 \times 4800 \mathrm{~km}^{2}$, corresponding to $6.6^{\prime \prime} \times 6.6^{\prime \prime}$ and spans a height range of $2800 \mathrm{~km}$ from the top layers of the convection zone to the middle chromosphere. Details of the simulation, carried out with the $\mathrm{CO}^{5} \mathrm{BOLD}$ code, can be found in Schaffenberger et al. (2005, 2006).

Initially, a homogeneous vertical magnetic field with a strength of $1 \mathrm{mT}$ was superposed on a model of relaxed thermal convection. After relaxation, fields of mixed polarity occur throughout the photosphere with an area-fractional imbalance typically of 3:1 for fields stronger than $1 \mathrm{mT}$. Similar polarity imbalances of even larger fields of view also occur in observational data (Lites 2002). Because of the periodic side boundaries and the conditions that the magnetic field must remain vertical at the top and bottom boundaries, the horizontally averaged vertical net magnetic-flux density remains $1 \mathrm{mT}$ throughout the simulation. This value compares very well with the mean flux density of $1.12 \mathrm{mT}$ reported by Lites et al. (2007a) for the Hinode data set. The mean absolute horizontal field strength is $2.5 \mathrm{mT}$ in the upper photosphere of the simulation, 2.5 times the net vertical flux density. Lites et al. (2007a) report a ratio of 5.

The grid constant of the computational domain in the horizontal direction is $0.055^{\prime \prime}\left(120^{3}\right.$ grid cells), three times smaller than the spatial sampling of the Hinode SP data. The simulation was advanced for $4400 \mathrm{~s}$ of real time. The results presented here refer to the last 2400 s, well after the initial relaxation phase. Using the SIR code (Ruiz Cobo \& del Toro Iniesta 1992; Bellot Rubio 2003), we synthesized the Stokes profiles of the Fe I $630 \mathrm{~nm}$ line pair from a large number of simulation snapshots with a spectral sampling of $2 \mathrm{pm}$. Profiles were computed along vertical lines-of-sight for each grid point. To the resulting intensity maps, we additionally applied a theoretical point-spread function (PSF) that models the spatial resolution capability of the SOT optics (Wedemeyer-Böhm 2008).

\section{Data analysis}

The line parameters extracted from both the calibrated Stokes data of the observation and from the simulation include the area and amplitude asymmetry of Stokes- $V$, the line-core and linewing velocities, and the total (signed) circular polarization

$V_{\text {tot }}=\int_{\lambda_{\mathrm{b}}}^{\lambda_{0}} V(\lambda) / I_{\mathrm{c}} \mathrm{d} \lambda-\int_{\lambda_{0}}^{\lambda_{\mathrm{r}}} V(\lambda) / I_{\mathrm{c}} \mathrm{d} \lambda$,

where $\lambda_{0}$ is the zero-crossing wavelength of a regular Stokes- $V$ profile, and $\lambda_{\mathrm{r}}$ and $\lambda_{\mathrm{b}}$ denote fixed wavelengths in the red and blue continua of the lines (similar to Rezaei et al. 2007). The relative Stokes- $V$ area asymmetry (Solanki \& Stenflo 1984) is defined as

$\delta A=\left(\int_{\lambda_{\mathrm{b}}}^{\lambda_{0}}|V(\lambda)| \mathrm{d} \lambda-\int_{\lambda_{0}}^{\lambda_{\mathrm{r}}}|V(\lambda)| \mathrm{d} \lambda\right) / \int_{\lambda_{\mathrm{b}}}^{\lambda_{\mathrm{r}}}|V(\lambda)| \mathrm{d} \lambda$.

Figure 1 shows a few selected examples of magnetic elements from the Hinode data (Cols. a-c) and the simulation (Cols. d-g). The observed ones are all taken from network cell interiors. Columns e and $g$ are based on the same synthetic data as are columns $\mathrm{d}$ and $\mathrm{f}$, respectively, but degraded with the theoretical PSF for SOT. The top row displays the sign of the area asymmetry: black corresponds to negative and white to positive area asymmetries. Gray indicates that the Stokes- $V$ profile has a signal below the $3 \sigma$ noise level ( $65 \%$ of the total field of view) or that it has an irregular shape $(20 \%)$, where most of the latter would classify as regular when lowering the noise level. This same threshold also applies to the simulation data of the panels 

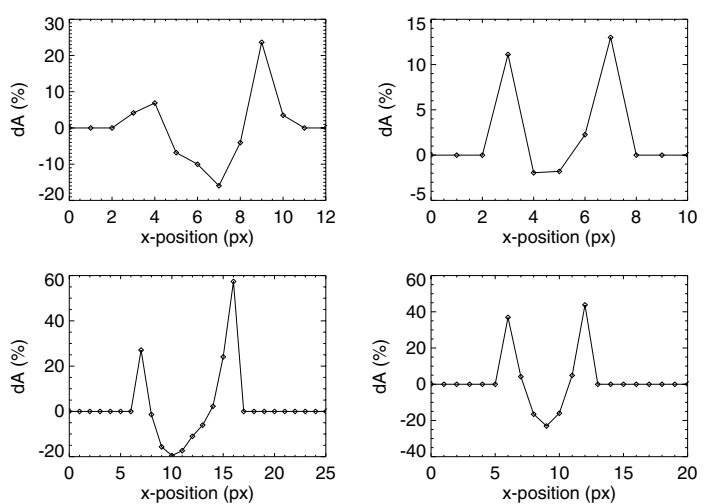

Fig. 2. Variation in $\delta A$ across magnetic elements from the Hinode data (top row) and the simulation (bottom row). From left to right and top to bottom, the sections are taken from the data corresponding to Cols. a, b, d, and f of Fig. 1.

in columns d-g, where we ignore $V$-profiles with an amplitude less than $3.6 \times 10^{-3} I_{\mathrm{c}}$ for comparison with the Hinode data. In the rightmost two panels, this threshold is 5 times lower in order to highlight the case of a weak magnetic element that would have barely been detected with Hinode.

The line-wing velocity of Fe I $630.15 \mathrm{~nm}$, shown in the second row, is obtained by averaging the Stokes- $I$ Doppler shift in the intensity interval $0.8-0.9 I_{\mathrm{c}}$. We set the convective blue shift of the line-core velocity in the quiet Sun to $-0.2 \mathrm{~km} \mathrm{~s}^{-1}$. Because of the low degree of polarization, the line-wing velocity is not spoilt by magnetic influence.

The third row displays $V_{\text {tot }}$, whose contours are shown in all the panels. It is a linear measure of the magnetic flux density according to Lites et al. (1999) and Lites et al. (2007b). From the fourth row, which shows the continuum intensity at $630 \mathrm{~nm}$, we see that the magnetic flux concentration occurs mainly in the intergranular space.

\section{Results and discussion}

Both the observation and the simulation show magnetic elements of either sheet like or roundish shape, possessing the same striking pattern with regard to the Stokes- $V$ asymmetry: a central region with negative Stokes- $V$ area asymmetry is framed or surrounded by a narrow seam of pixels having positive area asymmetry (Fig. 1, first row). Plotting $\delta A$ across a magnetic element, we find that $|\delta A|$ is typically larger in the periphery than in the central region of the magnetic flux concentration. Representative cross sections that demonstrate this behaviour are shown in Fig. 2 for both the observation and the simulation. Along with $\delta A$ in the top right panel of Fig. 2, Fig. 4 also gives the corresponding observed Stokes- $V$ profiles. Also from Fig. 1 we see that the magnetic elements are located within and surrounded by a downdraft.

For gaining deeper insight, we now consider in Fig. 3 the vertical cross section through the simulation snapshot shown in column d of Fig. 1 for the position $y=1.15^{\prime \prime}$. It displays the magnetic field strength (colour-coded) together with the velocity field, projected on the vertical plane (white arrows). The horizontally running curve indicates optical depth $\tau_{500 \mathrm{~nm}}=1$. A magnetic flux concentration is forming in the downdraft and has reached a field strength of $0.13 \mathrm{~T}$ at $\tau_{500 \mathrm{~nm}}=1$. Close to the surface of optical depth unity and through the photosphere, a relatively sharp, funnel-shaped boundary separates the magnetic flux concentration from the weak-field or field-free surroundings.

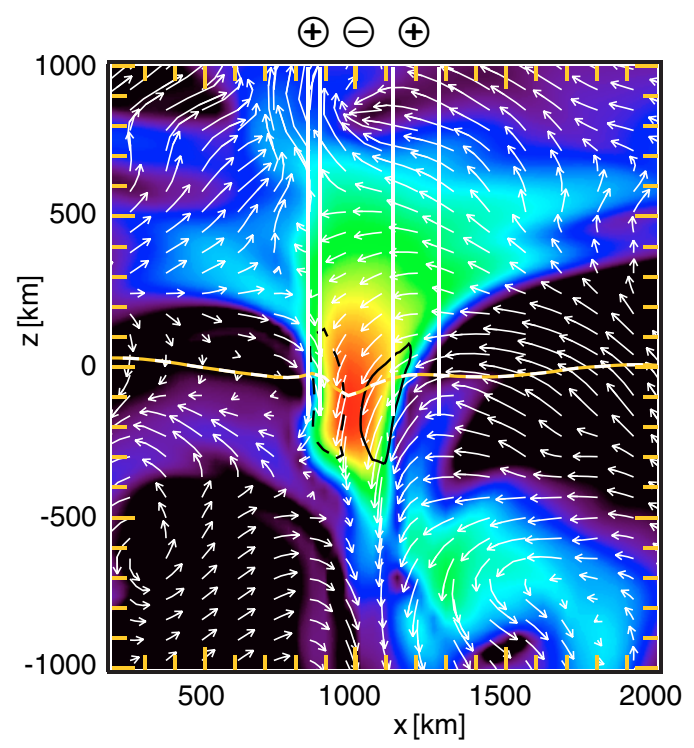

Fig. 3. Vertical cross section through the simulation box corresponding to position $y=1.15^{\prime \prime}$ of the column d of Fig. 1. It displays the logarithmic magnetic field strength (from 1.0 to $10^{2.3} \mathrm{mT}$, colour-coded), together with the velocity field, projected on the vertical plane (white arrows) and the electric current density normal to the plane (black contours). Optical depth $\tau_{500 \mathrm{~nm}}=1$ is shown as well. The white vertical lines indicate the ranges in which Stokes- $V$ profiles from vertical linesof-sight have either positive or negative area asymmetry, $\delta A$. Outside this range, the Stokes-V signal is below the $3 \sigma$ noise level of the observations. $\delta A(x)$ is plotted in the lower left panel of Fig. 2.

The inner vertical white lines indicate a central part of the flux concentration $(880<x<1120 \mathrm{~km})$, where Stokes- $V$ profiles have $\delta A<0$. This is because their lines-of-sight sample increasing field strength and increasing downflow velocity with increasing continuum optical depth (Solanki \& Pahlke 1988). This situation changes drastically in the peripheral region $840 \leq x<880 \mathrm{~km}$ and $1120<x \leq 1280 \mathrm{~km}$ (also indicated with white lines), where $\delta A>0$. Here, the downflow speed still increases with optical depth; but because of the funnel-shaped flux concentration, lines-of-sight traverse a magnetic boundary layer, where the field strength rapidly drops with increasing optical depth. The combination of increasing velocity and decreasing field strength produces positive area asymmetry. From this, we conclude that the narrow seam of positive area asymmetry in the periphery of magnetic flux elements seen in the Hinode data is a consequence of their well-defined boundary (or magnetopause) that expands with height in a funnel-like manner.

As an immediate consequence of Ampère's law, this boundary carries an electric current sheet. The black contours in Fig. 3 indicate a current density of $0.5 \mathrm{~A} \mathrm{~m}^{-2}$, encircling higher values of up to $4.0 \mathrm{~A} \mathrm{~m}^{-2}$. This refers to the current component perpendicular to the section plane, which flows in the opposite direction on either side of the magnetic flux concentration.

If the magnetic flux concentration is inclined enough with respect to the viewing direction, lines-of-sight traverse the boundary layer on only one side of the flux concentration, which leads to a one-sided seam of positive area asymmetry. This is almost the case in Fig. 3 (and corresponding $\delta A$-panel of Fig. 1), where the flux concentration is slightly inclined to the right making the seam of positive $\delta A$ to the left much narrower than that on the right side. In fact, most magnetic elements in the field of view of the observation show a one-sided pattern like in the upper right corner of the synthetic $\delta A$-map in Cols. $\mathrm{d}$ or e. 


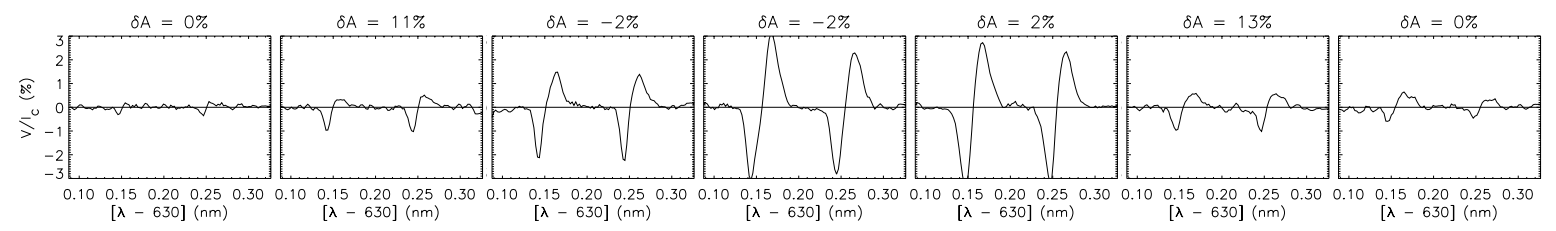

Fig. 4. Stokes- $V$ profiles from the Hinode data across the magnetic element of column b of Fig. 1. $\delta A(x)$ for the same section is shown in Fig. 2 (top right panel).

Because the mean magnetic flux density in the network cell interior is low, granular flow gathers only small amounts of flux so that only small and weak magnetic flux concentrations form (Steiner 2003). They form and disperse with the onset and cessation of intergranular downdrafts. Therefore we observe strong downflows associated with the magnetic elements considered in this work. Indeed, Grossmann-Doerth et al. (1996), Sigwarth et al. (1999), and Martínez Pillet et al. (1997) also observe mainly downflows associated with weak magnetic signals. In combination with increasing field strength, these downflows give rise to negative area asymmetry. Yet, the peripheral positive area asymmetry is larger (Fig. 2) and may partially balance or even outweigh the negative contribution when observing at a lower spatial resolution.

Langangen et al. (2007) finds that the downdraft velocity near the edges of magnetic elements is larger than in the central part. Here we find in both observations and simulation the maximum speed in the centre of the flux concentration. In the simulation this is because the magnetic element forms as a consequence of an intergranular downdraft, with peak velocity in the centre. This is different from the regime of a mature strong flux concentration for which simulations exhibit veritable downflow jets near their edges but not in the centre (Steiner et al. 1998; Shelyag et al. 2007). This regime might be more representative of the active region elements observed by Langangen et al. (2007).

Grossmann-Doerth et al. (1988, 1989) and Solanki (1989) first pointed to the peripheral, canopy-like magnetopause of magnetic elements as the origin of the observed positive Stokes- $V$ area asymmetry. Steiner (1999) found this effect at work in a two-dimensional MHD-simulation, but also found that the central region of the magnetic flux sheet tends to have negative values and that a delicate balance with the peripheral region exists in which the positive values may dominate and outweigh the negative ones. These findings have recently been confirmed in a three-dimensional environment by Shelyag et al. (2007). Bellot Rubio et al. (2000) came to a similar conclusion based on semi-empirical modelling. Leka \& Steiner (2001) found a similar pattern in the Stokes- $V$ area asymmetry in pores and magnetic knots. Here, for the first time, we find this pattern in observations of small-scale magnetic elements in the network cell interior.

\section{Conclusion}

Spectro-polarimetric data of a quiet Sun area close to the disc centre obtained with SOT on board Hinode were analysed. We find magnetic elements of either a roundish (tube) or an elongated (sheet) shape, which show Stokes- $V$ profiles of negative area asymmetry in the centre, surrounded or framed by pixels of larger, positive area asymmetry. A comparison with results from 3D MHD-simulations suggests that this peculiar pattern in Stokes- $V$ area asymmetry (first predicted by Steiner 1999) is due to the confined nature of the field in magnetic elements with a funnel-shaped boundary layer that gives rise to a steep gradient in field strength along the line of sight.
We also conclude that this kind of magnetic element of the internetwork is accompanied by electric current sheets. While these conclusions are evident from the comparison, we cannot exclude the hypothesis that a suitable magnetic structuring on scales not resolved by the present observation and simulation would lead to the observed pattern in $\delta A$.

Acknowledgements. Hinode is a Japanese mission developed and launched by ISAS/JAXA, with NAOJ as the domestic partner and NASA and STFC (UK) as international partners. It is operated by these agencies in co-operation with ESA and NSC (Norway). Part of this work was supported by the Deutsche Forschungsgemeinschaft (SCHM 1168/8-1).

\section{References}

Bellot Rubio, L. R. 2003, Inversion of Stokes profiles with SIR (Freiburg: Kiepenheuer Institut für Sonnenphysik)

Bellot Rubio, L. R., Ruiz Cobo, B., \& Collados, M. 2000, ApJ, 535, 489

Berger, T. E., Rouppe van der Voort, L. H. M., Löfdahl, M. G., et al. 2004, A\&A, 428,613

Grossmann-Doerth, U., Schüssler, M., \& Solanki, S. K. 1988, A\&A, 206, L37 Grossmann-Doerth, U., Schüssler, M., \& Solanki, S. K. 1989, A\&A, 221, 338 Grossmann-Doerth, U., Keller, C. U., \& Schüssler, M. 1996, A\&A, 315, 610 Ichimoto, K., Lites, B. W., Elmore, D., et al. 2007, Sol. Phys., submitted Illing, R. M. E., Landman, D. A., \& Mickey, D. L. 1975, A\&A, 41, 183 Khomenko, E. V., Martínez González, M. J., Collados, M., et al. 2005, A\&A, 436, L27

Kosugi, T., Matsuzaki, K., Sakao, T., et al. 2007, Sol. Phys., submitted Landolfi, M., \& Landi degl'Innocenti, E. 1996, Sol. Phys., 164, 191

Langangen, Ø., Carlsson, M., Rouppe van der Voort, L., \& Stein, R. F. 2007, ApJ, 655, 615

Leka, K. D., \& Steiner, O. 2001, ApJ, 552, 354

Lites, B. W. 2002, ApJ, 573, 431

Lites, B. W., Rutten, R. J., \& Berger, T. E. 1999, ApJ, 517, 1013

Lites, B. W., Kubo, M., Socas-Navarro, H., et al. 2007a, ApJ, submitted

Lites, B. W., Socas-Navarro, H., Kubo, M., et al. 2007b, PASJ, submitted

Martínez Pillet, V., Lites, B. W., \& Skumanich, A. 1997, ApJ, 474, 810

Rezaei, R., Schlichenmaier, R., Schmidt, W., \& Steiner, O. 2007, A\&A, 469, L9

Ruiz Cobo, B., \& del Toro Iniesta, J. C. 1992, ApJ, 398, 375

Sanchez Almeida, J., Collados, M., \& del Toro Iniesta, J. C. 1989, A\&A, 222, 311

Schaffenberger, W., Wedemeyer-Böhm, S., Steiner, O., \& Freytag, B. 2005, in Chromospheric and Coronal Magnetic Fields, ed. D. E. Innes, A. Lagg, \& S. A. Solanki, ESA SP, 596

Schaffenberger, W., Wedemeyer-Böhm, S., Steiner, O., \& Freytag, B. 2006, in Solar MHD Theory and Observations, ed. J. Leibacher, R. F. Stein, \& H. Uitenbroek, 345, ASP Conf. Ser., 354

Shelyag, S., Schüssler, M., Solanki, S. K., \& Voegler, A. 2007, A\&A, 467

Shimizu, T., Nagata, S., Tsuneta, S., et al. 2007, Sol. Phys., submitted

Sigwarth, M., Balasubramaniam, K. S., Knölker, M., \& Schmidt, W. 1999, A\&A, 349,941

Solanki, S. K. 1989, A\&A, 224, 225

Solanki, S. K., \& Pahlke, K. D. 1988, A\&A, 201, 143

Solanki, S. K., \& Stenflo, J. O. 1984, A\&A, 140, 185

Steiner, O. 1999, in Magnetic Fields and Oscillations, ed. B. Schmieder,

A. Hofmann, \& J. Staude, 38, ASP Conf. Ser., 184

Steiner, O. 2003, A\&A, 406, 1083

Steiner, O., Grossmann-Doerth, U., Knölker, M., \& Schüssler, M. 1998, ApJ, 495, 468

Suematsu, Y., Tsuneta, S., Ichimoto, K., et al. 2007, Sol. Phys., submitted

Tarbell, T. D. 2006, in AAS/Solar Physics Division Meeting, 7, 36

Tsuneta, S., Suematsu, Y., Ichimoto, K., et al. 2007, Sol. Phys., submitted Wedemeyer-Böhm, S. 2008, A\&A, in prep. 